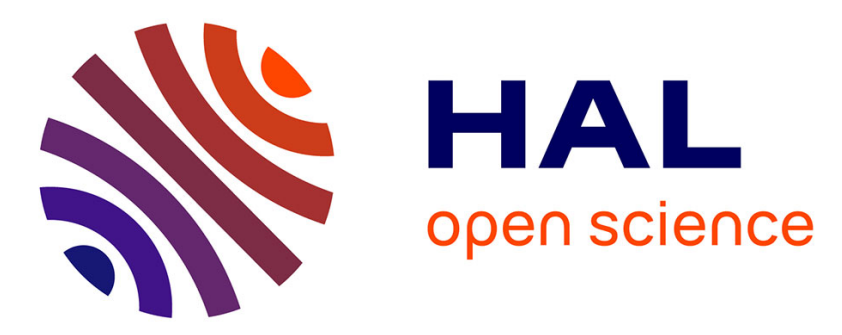

\title{
Attribute Profiles on Derived Textural Features for Highly Textured Optical Image Classification
}

\author{
Minh-Tan Pham, Sébastien Lefèvre, François Merciol
}

\section{To cite this version:}

Minh-Tan Pham, Sébastien Lefèvre, François Merciol. Attribute Profiles on Derived Textural Features for Highly Textured Optical Image Classification. IEEE Geoscience and Remote Sensing Letters, 2018, 15 (7), pp.1125-1129. hal-01672850

\section{HAL Id: hal-01672850 https://hal.science/hal-01672850}

Submitted on 14 Nov 2019

HAL is a multi-disciplinary open access archive for the deposit and dissemination of scientific research documents, whether they are published or not. The documents may come from teaching and research institutions in France or abroad, or from public or private research centers.
L'archive ouverte pluridisciplinaire HAL, est destinée au dépôt et à la diffusion de documents scientifiques de niveau recherche, publiés ou non, émanant des établissements d'enseignement et de recherche français ou étrangers, des laboratoires publics ou privés. 


\title{
Attribute profiles on derived textural features for highly-textured optical image classification
}

\author{
Minh-Tan Pham, Member, IEEE, Sébastien Lefèvre, François Merciol
}

\begin{abstract}
Morphological attribute profiles (APs) have been so far proved to be effective for remote sensing image classification by several research studies. However, recent studies have shown that a direct application of APs to highly textured and structured images, especially in very high resolution (VHR) optical imagery, may be insufficient. Some solutions have been proposed to deal with this issue such as to extract the local histograms and the local features of AP images (histogram-based APs, local feature-based APs, respectively); or to combine APs with different textural features. In this letter, we review these approaches and then propose a novel strategy which directly generates APs on some derived textural features, instead of separately combining them. Experimental results from both natural textures and VHR optical remotely sensed images show that the proposed approach can produce superior classification performance than the standard APs, HAPs, LFAPs as well as the classical combination of APs with textural features.
\end{abstract}

Index Terms-Remote sensing imagery, texture, morphological attribute profiles, supervised classification, random forest

\section{INTRODUCTION}

D ENSE pixel-level image classification for land use and land cover Earth Observation has been one of the most crucial tasks in remote sensing imagery and drawn lot of attention from researchers during past decades. Among a great number of proposed techniques in the literature, morphological attribute profiles (APs) [1] have been widely used thanks to their powerful multilevel modeling of spatial information from the image content and their efficient implementation via tree structures. By well preserving significant spatial properties of regions and objects such as contours, shape, etc., APs and their extensions (see a recent survey in [2]) become effective to characterize the contextual information of the observed scene, hence relevant for a classification purpose.

However, with the emergence of very high spatial spatial resolution (VHR) remote sensing technology, recent studies emphasize that a direct exploitation of APs for classification task may be insufficient for a complete characterization of structural and textural information from the image, especially when regions and objects become more heterogeneous in images acquired by such VHR sensors. Some efforts have been proposed to tackle this issue by using the local histogrambased APs (HAPs) [3] or the local feature-based APs (LFAPs) [4], [5]. These techniques propose to replace each AP sample response by the histogram or some first-order statistical features of the local patch around that AP's pixel position. They have been proved to be more efficient and better deal with

The authors are with the IRISA Lab, Universite Bretagne Sud, Vannes 56000, France. e-mail: minh-tan.pham@irisa.fr.

This work is funded by a Grant from Région Bretagne. local textures. Not focusing on improving the performance of APs, other studies have tried to combine APs with textural features yielded from popular techniques such as the GrayLevel Cooccurrence Matrix (GLCM), the Gabor features, etc. [6]-[8]. By employing ensemble classification as well as feature fusion techniques, they have reported some improvements compared to the separate use of morphological profiles or textural features.

Nevertheless, all of the above-mentioned techniques are still constraint to the fact that spatial AP features are generated directly from the original image. Since APs are derived from the pixel gray levels (i.e. by reconstruction from a tree), the appearance of structured textures may lead to the so-called chaining effect and hence reduce their performance. We will observe these phenomena in the next section of this letter. In this work, we focus on how to exploit and adapt the use of APs for VHR images with high level of structured textures. After reviewing the mentioned approaches, our motivation is to propose an effective way to overcome those problems by constructing trees (and then computing APs) not from the input image, but from some textural measurements from it. We prove that this approach can outperform the above existing methods and argue that depending on application, the use of APs should be adapted. In case of highly textured images, AP construction from derived textural features could benefit classification task.

The following section recalls the background of APs and points out their limitation when dealing with textures. The proposed strategy to generate APs on derived textural features is then described in Sec. III. In Sec. IV, we provide and discuss the results of our classification experiments. Section V finally concludes this work and addresses some prospective studies.

\section{BACKGROUND}

\section{A. APs}

APs are multilevel, efficient, and effective tools constructed from hierarchical representations of an input image [1]. Initially, it was generated based on a min-tree and a max-tree, but other implementations using the tree of shapes (i.e. selfdual APs) or the partition trees (such as $\alpha$-tree and $\omega$-tree to produce $\alpha$-profiles, $\omega$-profiles, respectively) [2] have been proposed. We note that throughout the paper, the standard APs is exploited to describe the proposed framework. However, the strategy is applicable to all above-mentioned trees.

As illustrated in Fig. 1, the generation of APs from an image can be summarized as a four-step process: 1) construct the tree from the image; 2) compute some relevant attributes (area, moment of inertia, standard deviation, etc.) from each node of 


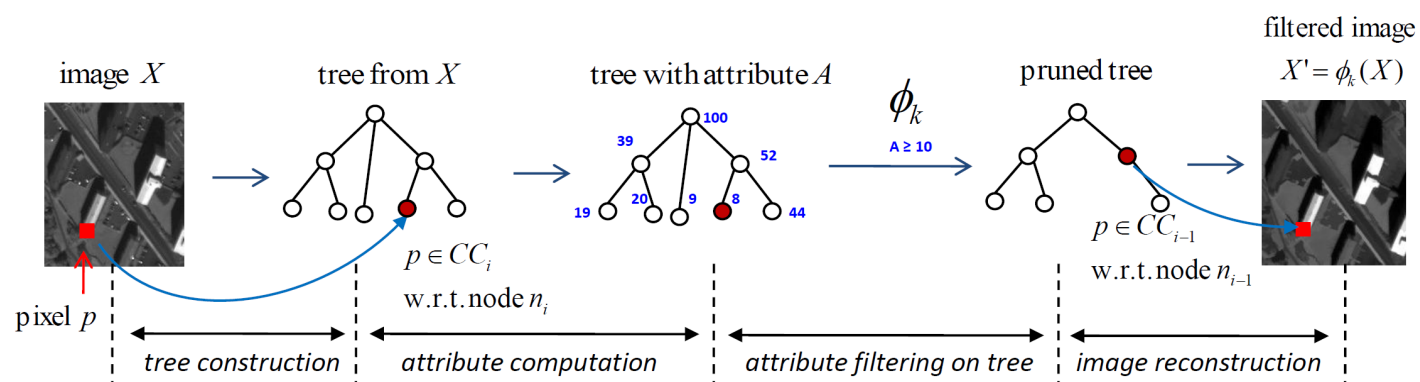

Fig. 1. AP generation framework from a grayscale image $X$ w.r.t the attribute filtering $\phi_{k}$.

the tree; 3 ) filter the tree by keeping/removing nodes according to their attribute values; 4) reconstruct the image from the filtered tree. Steps 3) and 4) can be done for different attributes (with different threshold values) to finally produce a set of filtered images (by stacking them) to form APs.

Formally, given a grayscale image $X: E \rightarrow \mathbb{Z}, E \subseteq \mathbb{Z}^{2}$, the standard generation of APs on $X$ is achieved by applying a sequence of AFs based on a min-tree (attribute thickening operators $\left.\left\{\phi_{k}\right\}_{k=1}^{K}\right)$ and a max-tree (i.e. attribute thinning operators $\left.\left\{\gamma_{k}\right\}_{k=1}^{K}\right)$ as follows:

$$
\begin{array}{r}
\operatorname{AP}(X)=\left\{\phi_{K}(X), \phi_{K-1}(X), \ldots, \phi_{1}(X), X,\right. \\
\left.\gamma_{1}(X), \ldots, \gamma_{K-1}(X), \gamma_{K}(X)\right\},
\end{array}
$$

where $\phi_{k}(X)$ is the filtered image obtained by the attribute thickening $\phi$ with regard to the threshold $k$. Similar explanation is made for $\gamma_{k}(X)$. The resulted $\operatorname{AP}(X)$ is a stack of $(2 K+1)$ images including the original image, $K$ filtered images from the thickening profiles and the other $K$ from the thinning profiles. In case of using the self-dual APs (SDAPs), the $\alpha$-APs or $\omega$-APs, the dimension of output profiles is reduced to $(K+1)$ since only one tree is exploited [2].

\section{B. Their Limitation When Dealing With Textures}

The effectiveness of APs is undeniable. However, when applying them to VHR remote sensing images where complex textural and structural information is relatively important, some limitations have been observed. In Figs. 2 and 3, we illustrate the chaining effect from the tree construction phase (here, the max-tree is used for illustration) which consequently appears within the output profiles (Fig. 3). In hierarchical image representation using tree structures, the chaining effect [9] is caused by the wrong connection of pixels, which are supposed to belong to one connected component dedicated to a thematic region or segment, to another connected component due to their close intensity levels. From Fig. 2, let us observe the structured texture on the top (forming by pixels of graylevels 1 and 3). During the max-tree construction, the dark part (gray-value 1) and the bright part (gray-level 3) have been separated and associated to different tree nodes. The bright part has been wrongly connected to the group of pixels of graylevel 4. As a result, when applying attribute filtering on trees constructed from real VHR images, the output filtered images usually involve several partitions (segments) suffering from the chaining effect. A clear example can be observed from
Fig. 3(b)-(c): many pixels from the vineyard areas (vertical oriented texture) are connected to the bright road, which will cause incorrect classification of these pixels (observed later in the experimental section).

\section{How TO EFFECTIVELY EXPLOIT APS FOR HIGHLY TEXTURED IMAGES?}

Section II-B has revealed the limitation of APs when applied to highly textural images (mainly caused by the chaining effect during the tree formation). The question is how to effectively exploit APs with such textural images. Some solutions have been proposed in the literature which can be divided into two main approaches: 1) extra spatial post-processing the APs; and 2) combining (by concatenating) the APs with some popular textural features. We review these related work and then propose our effective spatial-textural approach by performing APs on derived texture images.

\section{A. Related Studies}

1) HAPs and LFAPs: Recent studies including the local histogram-based APs (HAPs) [3] and the local features-based APs (LFAPs) [5] have focused on extra spatial processing of APs for better characterization of local texture information from remotely sensed images. In order to better deal with heterogeneous regions or objects (rich texture content), these papers proposed to replace each AP sample response (AP pixel value) by the histogram or some first-order statistical features of the local patch around that AP's pixel position. This extra post-processing technique helps to spatially smooth the output filtered images from the standard APs, which is relevant to tackle local texture description. As a result, HAPs and LFAPs have been proved to be more efficient than APs for image classification tasks [3], [5]. Nevertheless, when dealing with strong textural and structural features within VHR images (as in Fig. 3), they are still not able to compensate the chaining effect limitation within the AP computation.

2) APs+textural features: Another approach to exploit APs to tackle rich textural images is to combine them with popular textural measures such as the Haralick features, the Gabor or wavelet coefficients. Several researches in the literature have proposed to independently extract morphological or attribute profiles as well as textural features, and then concatenate them to form the final feature descriptor for classification [6][8]. This simple technique is a natural way to incorporate spatial and textural information from the image to enhance 


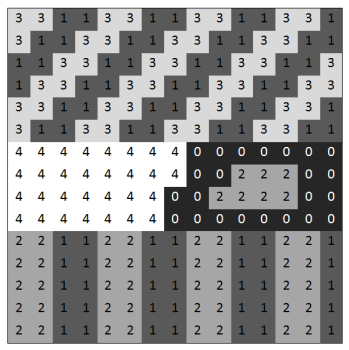

(a) Input image

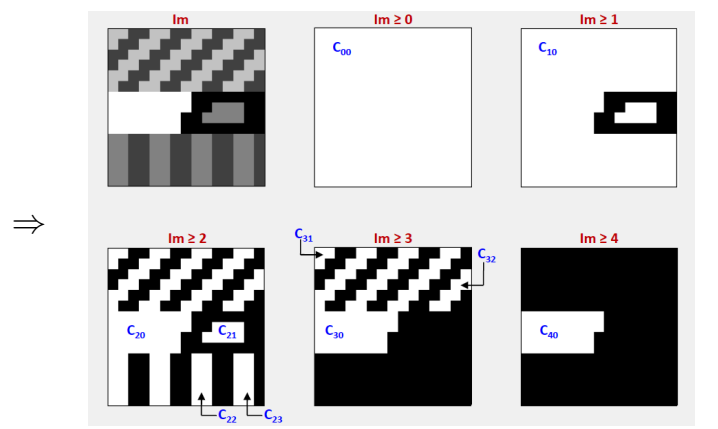

(b) Detailed construction

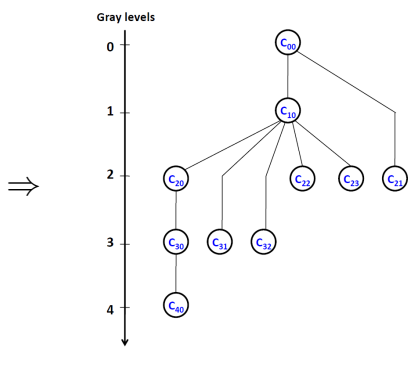

(c) Max-tree

Fig. 2. Chaining effect from the tree construction stage (i.e. a max-tree structure is exploited here for illustration).

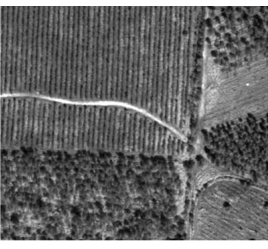

(a)

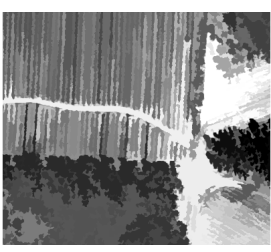

(b)

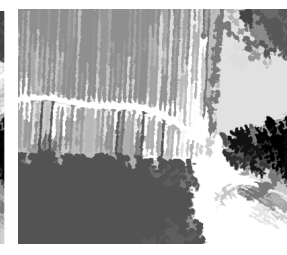

(c)
Fig. 3. Chaining effect which influences the output filtered images: (a) a VHR image crop; (b)-(c) two output images produced by attribute filtering with two different threshold values.

the classification performance. However, the drawback of APs applied to highly textured data has not been resolved since they were again generated directly from the original image.

\section{B. Proposed Approach: APs Derived From Textural Features}

The previous subsection has reviewed the two commonly used approaches exploiting APs for highly textural images. Although they can improve the classification performance compared to the standard APs, their results both still suffer from the influence of the chaining effect dedicated to the AP generation scheme itself. To overcome this, the main idea is to avoid generating APs directly from the original image, but from some image features which can ignore the impact of strong structural textures. In this work, we propose to compute APs from textural features instead of simply concatenating them as done by the approaches reviewed in Sec. III-A2.

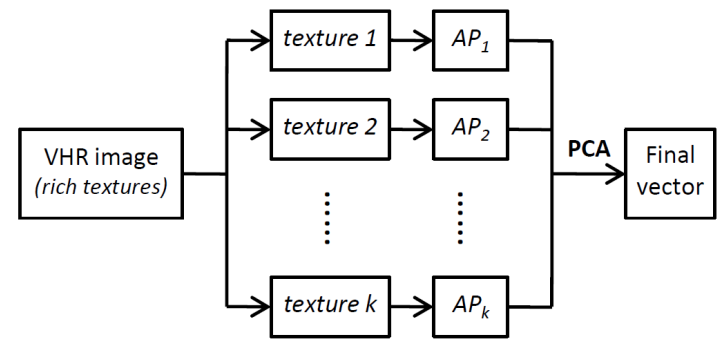

Fig. 4. Proposed framework to generate APs from different derived textural features applied to a highly textured VHR remotely sensed image.

The proposed technique is summarized in Fig. 4. Different texture signatures are first extracted from the input image. Each of them is then exploited to generate APs. Then, all the obtained APs are combined to form the final descriptor. Due to the issue of high dimensionality, the principle component analysis (PCA) is applied to reduce the feature dimension. We note that other feature extraction techniques (e.g. kernel PCA, ICA) can be applied at this stage but PCA is selected thanks to its popularity and stable performance.

Different types of textural characteristics can be utilized in the above scheme. In this letter, we exploit the Haralick features computed from the GLCM method [10]. The advantage of Haralick features is that they can encapsulate and characterize different texture properties: correlation, homogeneity, contrast, entropy, etc. By constructing APs from them, the proposed approach is able to model multilevel spatial information from a wide range of textural content. Despite its simplicity, the effectiveness of our approach is quite considerable. The next section will show readers its efficiency compared to related methods.

\section{EXPERIMENTAL STUDY}

\section{A. Datasets}

The main dataset in this work is a VHR panchromatic Pleiades image, distributed by Airbus DS/Spot Image, which has been used in [11]. The image (acquired in 2012) covers an intensively agricultural area near Tagon Marcheprime, France, with a $0.5-\mathrm{m}$ resampled resolution. The scene consists of different types of cultivation including parcels of vegetation, pine trees or vineyard areas, etc. Vines were planted in different row directions with various densities. There exist other types of texture classes: grass pasture, road and bare soil. These land cover characteristics introduce a variety of highly textured zones in the image. Experiments were conducted on a crop of $800 \times 1300$ pixels (Fig. 5(a)) with six classes including vine parcels in different row directions, pine, road and grass.

Besides, we also propose to employ the Brodatz texture database which is publicly available online to assess and validate the proposed method. An image of $1000 \times 1000$ pixels consisting of five different Brodatz textures (Fig.5(b)) was created and used for classification experiments. In fact, our purpose is to demonstrate the reliable behavior of the proposed approach for both widely used natural texture database as well as VHR optical remotely sensed images.

\section{B. Experimental Setup}

Supervised classification was performed on the two datasets using the random forest classifier with 200 trees. In the follow- 

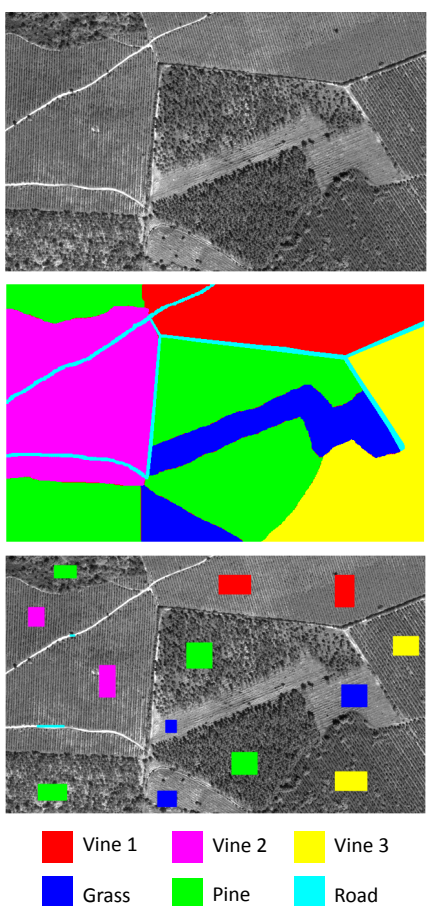

(a) VHR Pleiades image, (C)NES 2012

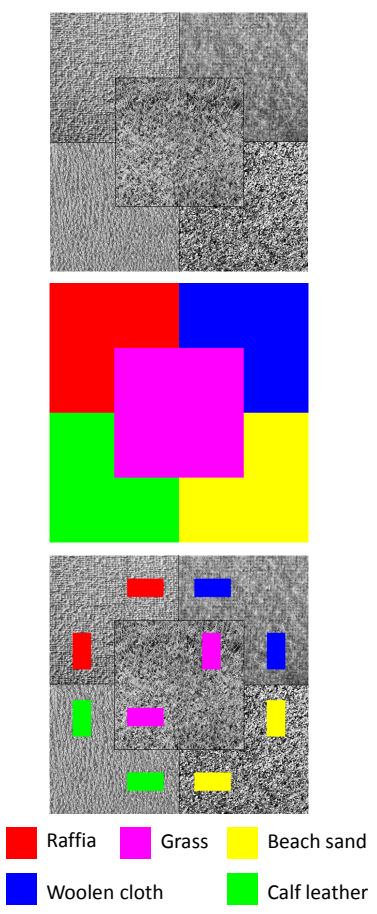

(b) Brodatz image

Fig. 5. Two data sets. From top to bottom: input image, ground truth, training sets (i.e. $5.8 \%$ of Pleiades pixels and $9.8 \%$ of Brodatz), class legends.

ing subsection, we report the classification results yielded by the proposed framework compared to several reference methods including: standard AP [1], self-dual AP [12], HSDAP [3], LFSDAP [5], GLCM [10], Gabor [13], SDAP+GLCM, SDAP+Gabor. Here, SDAPs were selected to perform most experiments thanks to their better performance and their lower dimension compared to standard APs [5], [12]. For attribute selection, we exploited the area, moment of inertia and standard deviation. Four thresholds (which were used in several AP-related papers e.g. [1], [5], [12], [14]) were set for each attribute: $\{100,500,1000,5000\}$ for area, $\{0.2,0.3,0.4,0.5\}$ for moment, and $\{20,30,40,50\}$ for standard deviation. For GLCM, 5 common Haralick measures were exploited including contrast, correlation, energy, homogeneity and entropy. Each one was extracted in 4 directions to create 20-D feature vector. Similarly, 20 Gabor features were extracted with 5 scales and 4 orientations. Both GLCM and Gabor were evaluated with 2 window sizes of $15 \times 15$ and $25 \times 25$ pixels.

For our approach, we performed and provided the results from 3 scenarios: SDAPs generated from all 5 Haralick measures (SDAP $\otimes$ GLCM), only from correlation texture $(\mathrm{SDAP} \otimes \mathrm{Corr})$ and only from homogeneity texture $(\mathrm{SDAP} \otimes$ Homo). Only GLCM features with window size $15 \times 15$ pixels were considered since our motivation is to prove that the method can provide good performance even with small GLCM window size. In addition, we also evaluated the proposed framework with and without applying the PCA in order to check its performance behavior. To this end, standard MATLAB implementation and equivalent parameter configuration of all methods were performed for a fair comparison.

TABLE I

CLASSIFICATION ACCURACY FOR THE TWO IMAGE DATA YIELDED BY DIFFERENT METHODS.

\begin{tabular}{l|c|cc|cc}
\hline \multirow{2}{*}{ Method } & \multirow{2}{*}{ Dim } & \multicolumn{2}{c}{ Pleiades } & \multicolumn{1}{c}{ Brodatz } \\
\cline { 3 - 6 } & & OA (\%) & Kappa & OA (\%) & Kappa \\
\hline AP & 27 & 67.22 & 0.5862 & 72.97 & 0.6673 \\
\hline SDAP & 15 & 68.42 & 0.5995 & 74.25 & 0.6774 \\
\hline HSDAP(nb=5) & 75 & 71.01 & 0.6489 & 78.04 & 0.7211 \\
\hline HSDAP(nb=7) & 105 & 73.13 & 0.6770 & 75.38 & 0.6895 \\
\hline LFSDAP & 30 & 75.86 & 0.6862 & 83.98 & 0.7986 \\
\hline GLCM15 & 20 & 79.18 & 0.7287 & 79.60 & 0.7447 \\
\hline GLCM25 & 20 & 81.61 & 0.7605 & 81.66 & 0.7699 \\
\hline Gabor15 & 20 & 78.34 & 0.7136 & 73.24 & 0.6653 \\
\hline Gabor25 & 20 & 76.92 & 0.6916 & 78.93 & 0.7356 \\
\hline SDAP+GLCM15 & 35 & 81.49 & 0.7625 & 84.28 & 0.8030 \\
\hline SDAP+GLCM25 & 35 & 84.26 & 0.7966 & 86.60 & 0.8322 \\
\hline SDAP+Gabor15 & 35 & 78.02 & 0.7184 & 80.66 & 0.7575 \\
\hline SDAP+Gabor25 & 35 & 81.93 & 0.7672 & 84.24 & 0.8272 \\
\hline \multicolumn{7}{|c|}{ Proposed approach without PCA } \\
\hline SDAP $\otimes$ GLCM15 & 300 & $\mathbf{8 8 . 3 1}$ & $\mathbf{0 . 8 4 8 5}$ & 91.92 & 0.8986 \\
\hline SDAP $\otimes$ Homo15 & 60 & 85.56 & 0.8129 & 88.38 & 0.8546 \\
\hline SDAP $\otimes$ Corr15 & 60 & 87.64 & 0.8402 & 90.53 & 0.8815 \\
\hline \multicolumn{7}{|c|}{ Proposed approach with PCA } & \\
\hline SDAP $\otimes$ GLCM15 & 20 & 86.73 & 0.8263 & $\mathbf{9 2 . 6 0}$ & $\mathbf{0 . 9 0 7 3}$ \\
\hline SDAP $\otimes$ Homo15 & 20 & 85.02 & 0.8025 & 89.42 & 0.8675 \\
\hline SDAP $\otimes$ Corr15 & 20 & 87.06 & 0.8297 & 90.63 & 0.8827 \\
\hline
\end{tabular}

\section{Results}

Table I reports the classification results of the two datasets in terms of overall accuracy (OA) and kappa coefficient. For both images, due to the high texture content, we observe poor performance from the standard AP as well as the SDAP. Next, HSDAP (with 2 different numbers of histogram bins) and LFSDAP have provided a slight improvement, but not good enough without the incorporation of textural features. The most effective reference approach is to combine SDAPs with GLCM $(25 \times 25$ pixels $)$ giving an OA of $84.26 \%$ for Pleiades and $86.60 \%$ for Brodatz. Then, by using the proposed method, we obtained the best performance of $88.31 \%$ for Pleiades and $92.60 \%$ for Brodatz, with a gain of $4.05 \%$ and $6.0 \%$, respectively. Here, it is worth noting that the proposed method based on GLCM of window $15 \times 15$ pixels could still significantly outperform SDAP+GLCM at larger window of $25 \times 25$ pixels. As another remark, we observe that by performing PCA on our approach (i.e. preserving 20 PCs), the classification accuracy has been slightly decreased for Pleiades, but slightly increased for Brodatz. A slight decrease in classification performance is caused by the information loss during feature reduction. Meanwhile, a slight increase may be resulted by a better combination and interaction of PCA features compared to the original ones. Nevertheless, all those slight changes are not too significant. By applying PCA for dimensionality reduction, the proposed approach still provides best results for both datasets.

For a more precise evaluation, Fig. 6 shows the classification maps obtained by the proposed approach compared with reference ones. As previously discussed in Sec. II-B, we observe the strong chaining effect phenomenon within the result of SDAP (Fig. 6(b)). Then, for other classical SDAPbased methods including HSDAP, LFSDAP, SDAP+GLCM and SDAP+Gabor (Fig. 6(c)-(d)-(h)-(i)), their results are 


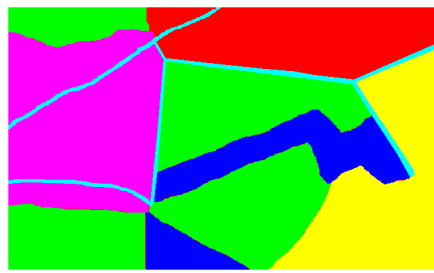

(a) Ground truth

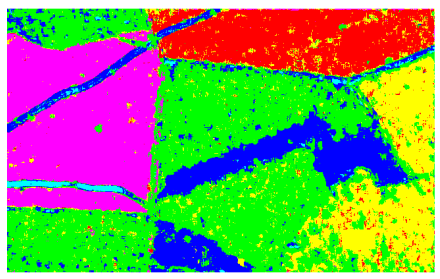

(e) GLCM15

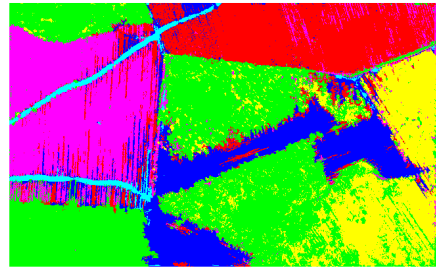

(i) SDAP+Gabor 15

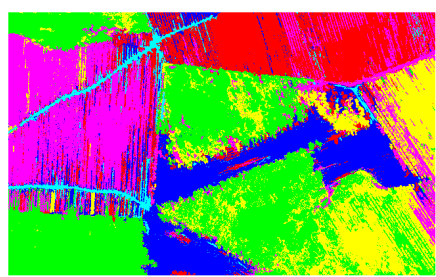

(b) SDAP

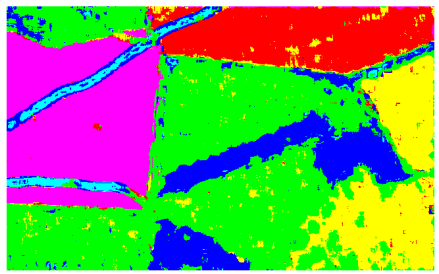

(f) GLCM25

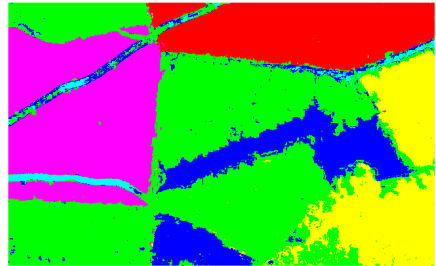

(j) $\mathbf{S D A P} \otimes$ GLCM15

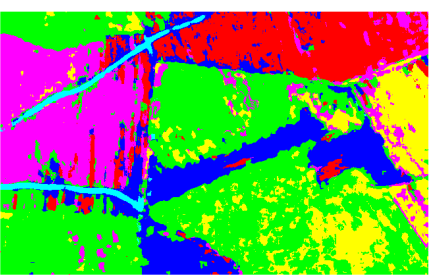

(c) $\operatorname{HSDAP}(\mathrm{n}=7)$

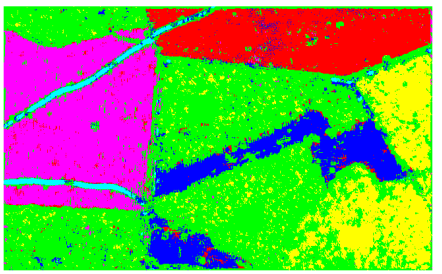

(g) Gabor15

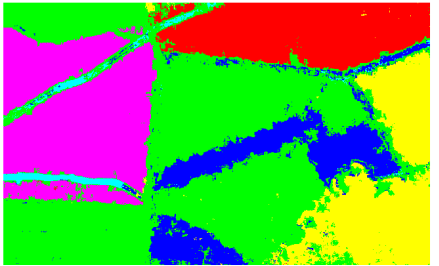

(k) SDAP $\otimes$ Homo15

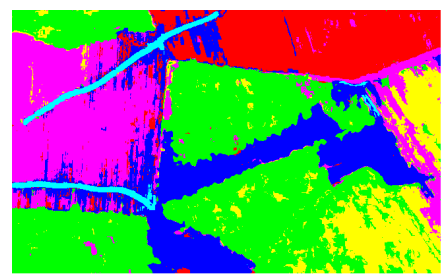

(d) LFSDAP

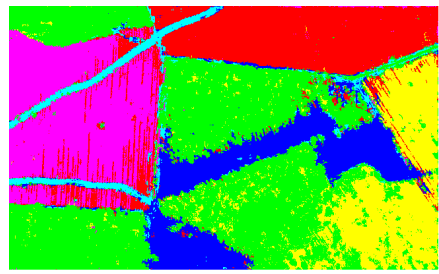

(h) AP+GLCM15

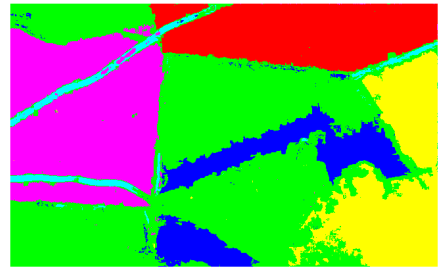

(1) $\mathbf{S D A P} \otimes$ corr15

Fig. 6. Classification maps of the VHR Pleiades image provided by the proposed approach (j-k-1) compared to reference methods (from $b$ to $i$ ).

smoother but still involve some wrongly classified red strips or blue strips within the magenta field. Those may be caused because SDAP features were modified (by histogram or local feature generation) or mixed together with GLCM/Gabor features, wrongly influencing on the classification process. The proposed method provided good classification maps (Fig. 6(j)(1)) even by exploiting only one Haralick measure such as homogeneity or correlation. To this end, the experimental results in Tab. I and Fig. 6 strongly validate our motivation and confirm the effectiveness of the proposed framework.

\section{CONCLUSION}

This letter has proposed an effective framework to apply attribute profiles to highly textured optical images. We have studied the limitation of direct AP exploitation which comes from the chaining effect phenomenon occurring during the tree formation. Classical approaches such as extra spatial processing or simply feature concatenation have been proved to be insufficient to deal with the problem. By generating APs from some derived textural features (e.g. Haralick features in this work), the proposed framework can better model rich texture image scenes and therefore provide superior classification results compared to reference methods. Future work may focus on exploiting alternative derived features as well as performing an automatic learning using deep neural networks.

\section{REFERENCES}

[1] M. Dalla Mura, J. A. Benediktsson, B. Waske, and L. Bruzzone, "Morphological attribute profiles for the analysis of very high resolution images," IEEE Trans. Geosci. Remote Sens., vol. 48, no. 10, pp. $3747-$ 3762,2010
[2] M.-T. Pham, S. Lefèvre, E. Aptoula, and L. Bruzzone, "Recent developments from attribute profiles for remote sensing image classification," in ICPRAI, 2018.

[3] B. Demir and L. Bruzzone, "Histogram-based attribute profiles for classification of very high resolution remote sensing images," IEEE Trans. Geosci. Remote Sens., vol. 54, no. 4, pp. 2096-2107, 2016.

[4] M.-T. Pham, S. Lefèvre, E. Aptoula, and B. B. Damodaran, "Classification of VHR remote sensing images using local feature-based attribute profiles," in IGARSS, 2017, pp. 747-750.

[5] M.-T. Pham, S. Lefèvre, and E. Aptoula, "Local feature-based attribute profiles for optical remote sensing image classification," IEEE Trans. Geosci. Remote Sens., vol. 56, no. 2, pp. 1199-1212, 2018.

[6] X. Huang and L. Zhang, "An SVM ensemble approach combining spectral, structural, and semantic features for the classification of highresolution remotely sensed imagery," IEEE Trans. Geosci. Remote Sens., vol. 51, no. 1, pp. 257-272, 2013.

[7] F. Mirzapour and H. Ghassemian, "Improving hyperspectral image classification by combining spectral, texture, and shape features," Int. J. Remote Sens., vol. 36, no. 4, pp. 1070-1096, 2015.

[8] L. Zhang, L. Zhang, D. Tao, and X. Huang, "On combining multiple features for hyperspectral remote sensing image classification," IEEE Trans. Geosci. Remote Sens., vol. 50, no. 3, pp. 879-893, 2012.

[9] P. Soille, "Constrained connectivity for hierarchical image partitioning and simplification," IEEE Trans. Patt. Anal. Mach. Intell., vol. 30, no. 7, pp. 1132-1145, 2008.

[10] R. M. Haralick et al., "Textural features for image classification," IEEE Trans. Sys. Man Cybern., vol. 3, no. 6, pp. 610-621, 1973.

[11] M.-T. Pham, G. Mercier, and J. Michel, "PW-COG: An effective texture descriptor for VHR satellite imagery using a pointwise approach on covariance matrix of oriented gradients," IEEE Trans. Geosci. Remote Sens., vol. 54, no. 6, pp. 3345-3359, 2016.

[12] M. Dalla Mura, J. Benediktsson, and L. Bruzzone, "Self-dual attribute profiles for the analysis of remote sensing images," in ISMM, 2011, pp. 320-330.

[13] A. K. Jain and F. Farrokhnia, "Unsupervised texture segmentation using Gabor filters," Patt. Recog., vol. 24, no. 12, pp. 1167-1186, 1991.

[14] M.-T. Pham, E. Aptoula, and S. Lefèvre, "Feature profiles from attribute filtering for remote sensing image classification," IEEE J. Sel. Topics Appl. Earth Observ. Remote Sens., vol. 11, no. 1, pp. 249-256, 2018. 